\title{
Error-Resistant Distributed Quantum Computation in Trapped Ion Chain
}

\author{
Sibylle Braungardt ${ }^{1}$, Aditi Sen $(\mathrm{De})^{1}$, Ujjwal Sen ${ }^{1}$, and Maciej Lewenstein ${ }^{2}$ \\ ${ }^{1}$ ICFO-Institut de Ciències Fotòniques, Mediterranean Technology Park, 08860 Castelldefels (Barcelona), Spain \\ ${ }^{2}$ ICREA and ICFO-Institut de Ciències Fotòniques, \\ Mediterranean Technology Park, 08860 Castelldefels (Barcelona), Spain
}

\begin{abstract}
We consider experimentally feasible chains of trapped ions with pseudo-spin $1 / 2$, and find models that can potentially be used to implement error-resistant quantum computation. Similar in spirit to classical neural networks, the error-resistance of the system is achieved by encoding the qubits distributed over the whole system. We therefore call our system a quantum neural network, and present a quantum neural network model of quantum computation. Qubits are encoded in a few quasi-degenerated low energy levels of the whole system, separated by a large gap from the excited states, and large energy barriers between themselves. We investigate protocols for implementing a universal set of quantum logic gates in the system, by adiabatic passage of a few low-lying energy levels of the whole system. Naturally appearing and potentially dangerous distributed noise in the system leaves the fidelity of the computation virtually unchanged, if it is not too strong. The computation is also naturally resilient to local perturbations of the spins.
\end{abstract}

\section{INTRODUCTION}

Quantum computers, if realized in laboratory, are known to be capable of solving problems much faster than classical computers. Two famous examples are the Shor algorithm [1] for factoring a nonprime integer $N$ in polynomial time in the number of binary digits of $N$, and the Grover algorithm [2], which can find a single object from an unsorted database of $N$ objects in an order of $\sqrt{N}$ calls to the database in a quantum computer. While the latter task requires an order of $N$ calls to the database in a classical computer, the former is strongly believed to require exponentially large time in the same.

One of the most challenging problems that occur when trying to build a quantum computer is decoherence. The system interacts with its environment, and the quantum logical gates cannot be implemented perfectly. A number of schemes for protecting quantum information have been developed, including fault tolerance codes [3], decoherence free subspaces [4], noiseless subsystems [5], dynamic decoupling [6], topological quantum computation [7], and geometric quantum computation [8].

Our approach to error resistant quantum computation is based on the idea of neural networks, which, classically, can offer robust (i.e. noise resistant) storage and manipulation of classical data by encoding the classical memory patterns in a distributed way in the whole neural network (see e.g. 9]). A typical classical neural network has a large number of metastable energy minima with large basins of attraction, which can be used for this purpose. A classical neural network is also typically characterized by long range interactions. Moreover, these interactions are usually disordered and "frustrated". The disordered interactions are motivated by realistic situations: The bonds that carry information between neurons in a brain are typically quite irregular, and fluctuate. Such disordered interactions have the effect that the different metastable energy minima are statistically independent, so that for large systems, their overlaps vanish.
"Frustration" in a network can be defined as a situation, where one cannot find a configuration of the "particles" (that make up the network) by satisfying all the interactions (bonds) between them. While there are physical (or biological) reasons for considering frustrated interactions, it is also (believed to be) important for having a large number of low lying metastable and "orthogonal" (in the sense of Hamming distance (see e.g. 10])) energy patterns.

Just as distributed classical information encoding in classical neural networks is good for classical data manipulation, we show that distributed quantum information encoding in their quantum analogs (we call them "quantum neural networks" (QNN)) can potentially be used for robust manipulation of quantum data: error resistant quantum computation. The system that we have in our minds for a possible implementation of the protocols that we describe in this paper, are systems of cold ions in a trap (see [13, 14, 15] and references therein). The state-of-the-art of current experiments (see e.g. 11, 12], and references therein) show, that such systems allow for a high degree of control of the parameters, and in particular, of the interactions. Consequently, in such systems, we are able to manipulate strictly orthogonal (in the usual sense of orthogonality of pure states in a Hilbert space) states of the whole system, without making use of disordered interactions. Moreover, this is possible with a mesoscopic number of ions in the system.

We propose to encode quantum data in the energy levels of the system, and perform quantum gates by adiabatic passage of these levels. Thus, a too large number of low lying energy levels will typically be detrimental for our purposes: the finer an avoided crossing is, the larger is the probability of the system to leak into higher excitations. Therefore, we also do not want frustration effects to dominate in our system and produce such low energy states.

Using such a quantum neural network, we show that one can implement not only one-qubit gates, but also universal two-qubit gates in a naturally error resistant 
way. The idea of the gate implementations is the following. Suppose that a (unitary) gate is defined as a transfer of an initial orthogonal set of vectors into a final one. We choose the initial parameters of the Hamiltonian of the system in such a way, that the initial orthogonal set of vectors can be encoded onto a few lower eigenstates of the initial Hamiltonian. Subsequently, the system parameters are changed (slowly, i.e. adiabatically), such that the final orthogonal set of vectors of the unitary gate, turns out be (approximately) the corresponding lower instantaneous eigenstates of the final Hamiltonian. The change in the Hamiltonian is brought about by the changing of certain external (parallel and transverse) fields, and these are the sole (external) parameters that needs to be changed for the adiabatic passage.

In a certain sense, our system resembles systems with topological order and topologically protected qubits, such as proposed in Ref. 7]. In those systems there exist several degenerated ground states that are separated from the excited states by a large energy gap and which are used to store the qubits. Local perturbations of such states are very insufficient: one has to go to very high orders of the perturbation theory in order to transfer one of the protected states into another, i.e. change the topological charge.

In our case, the situation is rather similar for most practical purposes. As we showed in Ref. [16], we can control the ion trap potential in such a way that the system has a quasi-degenerate low energy manifold. As the classical analysis in our earlier Letter (Ref. [16]) shows, all of these states are local minima of energy, which are stable with respect to multiple spin flips, just as in standard classical neural networks. We therefore have a number of states that are separated by a large gap from the higher excited states, and are separated between themselves by large barriers. This implies that also in our case, local perturbations are inefficient. The system (QNN) is thus intrinsically robust to local noise for quantum computational purposes - qubit states, when slightly perturbed locally, transform under gate operations, almost similarly as ideal qubit states. On top of that, the system exhibits even a different mechanism of error resistance, as we shall see below. In constructing the QNN, in a natural manner one obtains noise terms, which have a distributed character: they act globally on the whole system. Such terms are potentially dangerous and may seriously diminish the performance of the model. Fortunately, also in this case, energy gaps and barriers assure protection.

The paper is organized as follows. In Sect. III we briefly describe the adiabatic theorem. In Sect. III we give a description of the model of our QNN and introduce our noise models: local perturbations and the naturally appearing distributed noise. The quantum neural network model of quantum computation consists of two main steps: The distributed encoding of the qubits, and the implementation of the quantum gates by adiabatic passage. The encoding of the qubits is described in Sect.
IV] Sect. V defines the two gates, namely the $\mathcal{H}$ gate and the Bell gate, whose protocols for implementation are presented in Sect. VI. Sect. VII contains the resulting fidelities of the gates. In Sect. [VIII] we apply the adiabaticity condition to our system, and give constraints on the time of the evolution. We discuss our results in Sect. IX.

\section{THE ADIABATIC THEOREM}

The quantum adiabatic theorem [17, 18] states that a physical system that is initially in one of its nondegenerate eigenstates will remain in the corresponding instantaneous eigenstate, provided that the Hamiltonian is varied "sufficiently" slowly.

The time evolution of the system is given by the time dependent Schrödinger equation

$$
i \hbar \frac{d}{d t}|\Psi(t)\rangle=H(t)|\Psi(t)\rangle
$$

where we let our system evolve from $t=0$ until the time $t=T$. If we scale the time evolution by introducing a scale factor $s=\frac{t}{T}$, where $0 \leq s \leq 1$, the Schrödinger equation becomes

$$
i \hbar \frac{d}{d s}|\Psi(s)\rangle=T H(s) \Psi(s) .
$$

The time evolution of the system is described completely by the Hamiltonian and the initial state. The development of the system is considered as "adiabatic", so that the adiabatic theorem holds, if the change of the Hamiltonian is small as compared to the gap $g(s)$ between the energy levels; more precisely, if

$$
T \gg \hbar \frac{\left\|\frac{d}{d s} H(s)\right\|}{g(s)^{2}},
$$

where $\|\Lambda\|$ is the operator norm of $\Lambda$, defined as the square root of the maximal eigenvalue of $\Lambda^{\dagger} \Lambda$. If one desires to adiabatically transport the $i$ th eigenstates at a certain time to the $i$ th eigenstate at a different time, the gap $g(s)$ is the minimum of the energy gaps to the $(i-1)$ th and the $(i+1)$ th energy levels. If the adiabaticity condition is fulfilled, an evolution starting out in the $i$ th eigenstate of $\mathrm{H}(0)$ will end up, at time $t=T$, with high probability, in the $i$ th eigentstate of the Hamiltonian $H(T)$.

In this paper, adiabatic transport of superpositions of a few energy levels is considered. For a superposition of say the 2nd and the 3rd levels, the gap $g(s)$ is the minimum of the gaps between 1 st and 2 nd levels, 2 nd and 3rd levels, and 3rd and 4th levels. When applying the adiabatic theorem to superpositions of eigenstates, the phases are also relevant to the calculations. For example, a superposition

$$
a|2(0)\rangle+b|3(0)\rangle
$$


of the 2nd energy level $|2(0)\rangle$ and the 3rd level $|3(0)\rangle$ of the Hamiltonian $H(0)$, will end up, at time $T$, with high probability, in the superposition

$$
a e^{i \Phi_{2}}|2(T)\rangle+b e^{i \Phi_{3}}|3(T)\rangle
$$

of the 2nd energy level $|2(T)\rangle$ and the 3rd level $|3(T)\rangle$ of the Hamiltonian $H(T)$. The phases $\Phi_{i}$ are given by the sums of the dynamical and Berry phases [18, 19, 20, 21] for the corresponding eigenstates. The dynamical phase is given by

$$
\Phi_{i}^{D}=-\int_{0}^{T} E_{i}(t) d t,
$$

and the Berry phase is defined as

$$
\Phi_{i}^{B}=i \int_{0}^{T}\left\langle E_{i}(t)\left|\frac{d}{d t}\right| E_{i}(t)\right\rangle d t .
$$

The instantaneous eigenvalues of the Hamiltonian $H(t)$ are denoted as $E_{i}(t)(i=0,1,2, \ldots)$, with $E_{0}(t)<$ $E_{1}(t)<E_{2}(t)<\ldots$, for all time $t$. The instantaneous $i$ th eigenstate is denoted as $\left|E_{i}(t)\right\rangle$. The ground state $\left|E_{0}(t)\right\rangle$ will also be denoted as $|G(t)\rangle$.

Since the work of Farhi and Gutmann [22] (see also 23, 24], and references therein), the adiabatic theorem has been used for quantum information processing, and has been called "adiabatic quantum computation". A methodological difference between the above set of works and the present paper, is that in their case, the system is always in the ground state, while our system is typically a superposition of a few lower excited levels along with the ground state. Among other things, this may affect the adiabaticity condition. Perhaps even more important differences are as follows:

(i) "Special purpose" Hamiltonian versus "universal" Hamiltonian: Adiabatic quantum computation typically considers a certain quantum algorithm, and depending on the algorithm, a certain Hamiltonian is considered. It was shown in Ref. 24] that the set of 2-local Hamiltonians is enough for this purpose. We, however, have a single quantum Hamiltonian (the QNN), that we will show below to be enough for all quantum algorithms, as our Hamiltonian implements universal gates (like the Bell gate, defined in Sect. (V), which can be applied to simulate arbitrary quantum algorithms. In this sense, the QNN Hamiltonian is a universal Hamiltonian for quantum computation.

(ii) Noise-resistance mechanism: Below, we will observe that quantum computing in a system described by the QNN Hamiltonian is resistant to noise, and this resistance is related to the fact that the system mimics a neural network: the quantum information is distributed in the eigenstates of the whole system. Resistance to noise in adiabatic quantum computation has apparently a different origin, as the typical Hamiltonians there, are not fully connected [24].

\section{THE QUANTUM NEURAL NETWORK HAMILTONIAN AND OUR NOISE MODELS}

In this paper we will consider a system of trapped spin$1 / 2$ particles with long range interactions, that are subject to slowly changing (in real time $(t)$ ) external magnetic fields. Such a system can be implemented with ions in a trap, where two internal states of each ion serve as the "up" and "down" states (denoted as $|\uparrow\rangle$ and $|\downarrow\rangle$ respectively) of the pseudo spin- $1 / 2$ particles (see Refs. [13, 14]). As shown in the above references, such a system offers a wide variety of spin models, which can be implemented by changing the system parameters. We are interested in long range Ising interactions. As shown in Refs. 16, 25], the Hamiltonian of the system depends crucially on the geometry of the external trap potential. For the case of a harmonic trap, the time dependent Hamiltonian of a system of eight spins can be approximated by

$$
\begin{array}{r}
H(t)=-\lambda\left[r_{1}\left(S_{z 1}+S_{z 2}+S_{z 3}+S_{z 4}\right)^{2}\right. \\
+r_{2}\left(\left(S_{z 1}+S_{z 2}\right)-\left(S_{z 3}+S_{z 4}\right)\right)^{2} \\
+r_{3}\left(\left(S_{z 1}-S_{z 2}\right)-\left(S_{z 3}+S_{z 4}\right)\right)^{2} \\
+A(t)\left(S_{x 1}+S_{x 2}+S_{x 3}+S_{x 4}\right) \\
\left.+B_{1}(t)\left(S_{z 1}+S_{z 2}\right)+B_{2}(t)\left(S_{z 3}+S_{z 4}\right)\right],
\end{array}
$$

where, typically, $r_{1}$ is much greater than $r_{2}$ and $r_{3}$. The $r_{i}$ corresponding to higher modes are even smaller and are thus neglected. Here

$$
S_{\alpha i}=\sigma_{2 i-1}^{\alpha}+\sigma_{2 i}^{\alpha}, \quad i=1,2,3,4,
$$

and $A, B_{1}$ and $B_{2}$ are external magnetic fields. The overall factor $\lambda$, which has the units of energy, in the Hamiltonian $H(t)$ has the effect of making the rest of the parameters in the Hamiltonian dimensionless. As we will show, such a system (i.e., one in which $r_{1} \gg r_{2}, r_{3}$ ) can be used for implementing one-qubit gates, but is apparently not suitable for two-qubit universal gates. However, for trap potentials of the form $|x|^{\gamma}$, with $\gamma \approx 0.5$, one obtains a situation where $r_{1} \approx r_{2} \gg r_{3}[16,25]$. The trap for which $\gamma=0.5$ may be called a fountain trap. We show below that this latter case can be used for implementing both one- qubit and two-qubit gates. The consideration of eight spins in our system is motivated by the number of spins that is currently viable in ion trap experiments (see e.g. [12]).

We will now discuss two possible sources of noise that act on the system. We first consider a "distributed noise", i.e. noise that arises globally in the system. Secondly, we will discuss a "local noise", i.e. noise that acts on the states of the system via local perturbations.

\section{A. Distibuted Noise}

The terms in the quantum neural network Hamiltonian $H(t)$ corresponding to $r_{1}, r_{2}$, and $r_{3}\left(r_{4}, r_{5}\right.$, etc. 
are neglected here) stem respectively from the first, second, and third (and further) vibrational modes of the trapped ions system, since the phonons are the carriers of interactions between the spins. Therefore, in the case when $r_{1} \approx r_{2} \gg r_{3}$, one can consider the $r_{3}$ (as well as $r_{4}, r_{5}$, etc., if present) term as a distributed, and thus potentially dangerous, noise in the system. This noise model is motivated by taking into account the following points:

(i) Increasing the effect of the third (and also higher) vibrational mode, which in the undisturbed case is much smaller than the first and second ones, covers inaccuracies in the trapping potential.

(ii) Moreover, decreasing the eigenfrequency of the third vibrational mode (i.e. increasing $r_{3}$ ) introduces a disturbance in the motion of the ions.

(iii) In addition, this introduces noise in the spin, as it is the phonon modes that are the carriers of interaction between the effective spins.

Similarly, in the case when $r_{1} \gg r_{2}, r_{3}$ (e.g. in the case of the harmonic trap), the $r_{2}$ term can be considered as a model of noise in the system.

\section{B. Local Noise}

We will now discuss our model for local noise that can potentially act on our system, by considering local perturbations to the states of the system. Let us note that the term "local noise" in this manuscript does not mean that the noise is local with respect to the spins (ions). It means that the noise is local in the configuration space of the system. We consider imperfections to the initial state of the system by superposing it with the states where one spin is flipped. For the "all down" state $\mid \downarrow \downarrow \downarrow \downarrow \downarrow \downarrow \downarrow \downarrow$, for example, we consider the transformation

$$
|\downarrow \downarrow \downarrow \downarrow \downarrow \downarrow \downarrow\rangle \rightarrow|\downarrow \downarrow \downarrow \downarrow \downarrow \downarrow \downarrow\rangle+\varepsilon\left|W_{8}\right\rangle
$$

as a model of noise. The output of the noise effect is not yet normalized. Here $\left|W_{8}\right\rangle$ is the eight spin W-state, defined as the normalized symmetric superposition of all states with one spin up and the rest spins down [26]. The parameter $\varepsilon$ is the strength of the perturbation. In Sect. VII. we will study the influence of this local noise on the gate fidelities.

\section{DISTRIBUTED ENCODING OF THE QUBITS}

As noted before, the quantum neural network model of quantum computation consists of two steps, beginning with an encoding of the qubits in a distributed way: The qubits are encoded, as we discuss now, as eigenstates of the whole neural network.
We assume that the Hamiltonian $H(t)$ changes in a continuous way from a certain initial value $H(0)$ at time $t=0$ to a certain final value $H(T)$ at time $t=T$. Note that the change in the Hamiltonian is brought about solely by changes in the fields. We choose the initial fields in the QNN Hamiltonian such that the ground state and the three lowest excited states at the initial time $t=0$ are respectively

$$
\begin{aligned}
|G(0)\rangle & =|\uparrow \uparrow \uparrow \uparrow \uparrow \uparrow \uparrow \uparrow\rangle, \\
\left|E_{1}(0)\right\rangle & =|\downarrow \downarrow \downarrow \downarrow \downarrow \downarrow \downarrow \downarrow\rangle, \\
\left|E_{2}(0)\right\rangle & =|\uparrow \uparrow \uparrow \uparrow \downarrow \downarrow \downarrow \downarrow\rangle, \\
\left|E_{3}(0)\right\rangle & =|\downarrow \downarrow \downarrow \downarrow \uparrow \uparrow \uparrow \uparrow\rangle .
\end{aligned}
$$

For implementing one-qubit gates, we will use the following encoding:

$$
\begin{aligned}
|0\rangle=|G(0)\rangle & =|\uparrow \uparrow \uparrow \uparrow \uparrow \uparrow \uparrow \uparrow\rangle, \\
|1\rangle=\left|E_{1}(0)\right\rangle & =|\downarrow \downarrow \downarrow \downarrow \downarrow \downarrow \downarrow \downarrow\rangle .
\end{aligned}
$$

The extreme left hand sides of the above equations denote the logical states of the qubit.

On the other hand, for two-qubit gates, we will encode one qubit in the first four spins and the other qubit in the remaining four spins:

$$
\begin{aligned}
|00\rangle=|G(0)\rangle & =|\uparrow \uparrow \uparrow \uparrow \uparrow \uparrow \uparrow \uparrow\rangle, \\
|11\rangle=\left|E_{1}(0)\right\rangle & =|\downarrow \downarrow \downarrow \downarrow \downarrow \downarrow \downarrow \downarrow\rangle, \\
|01\rangle=\left|E_{2}(0)\right\rangle & =|\uparrow \uparrow \uparrow \uparrow \downarrow \downarrow \downarrow \downarrow\rangle, \\
|10\rangle=\left|E_{3}(0)\right\rangle & =|\downarrow \downarrow \downarrow \downarrow \uparrow \uparrow \uparrow \uparrow\rangle .
\end{aligned}
$$

Again the extreme left hand sides of the above equations denote the logical states of the two qubits.

\section{THE $\mathcal{H}$ GATE AND THE BELL GATE}

We consider implementations of a one-qubit, as well as a two-qubit gate. The two-qubit gate is an entangling one, so that along with one-qubit gates, a universal set of quantum gates is formed [29]. The one-qubit gate, in the logical basis, is given by

$$
|0\rangle \rightarrow|+\rangle \equiv \frac{|0\rangle+|1\rangle}{\sqrt{2}}, \quad|1\rangle \rightarrow-|-\rangle \equiv-\frac{|0\rangle-|1\rangle}{\sqrt{2}} .
$$

Note that this transformation, which we call the $\mathcal{H}$ gate, is closely related to the Hadamard transformation that takes

$$
|0\rangle \rightarrow|+\rangle \text { and }|1\rangle \rightarrow|-\rangle \text {. }
$$

The two-qubit gate that we consider here acts as

$$
\begin{aligned}
|00\rangle \rightarrow\left|\phi^{+}\right\rangle & \equiv \frac{|00\rangle+|11\rangle}{\sqrt{2},} \\
|11\rangle \rightarrow-\left|\phi^{-}\right\rangle & \equiv-\frac{|00\rangle-|11\rangle}{\sqrt{2}},
\end{aligned}
$$




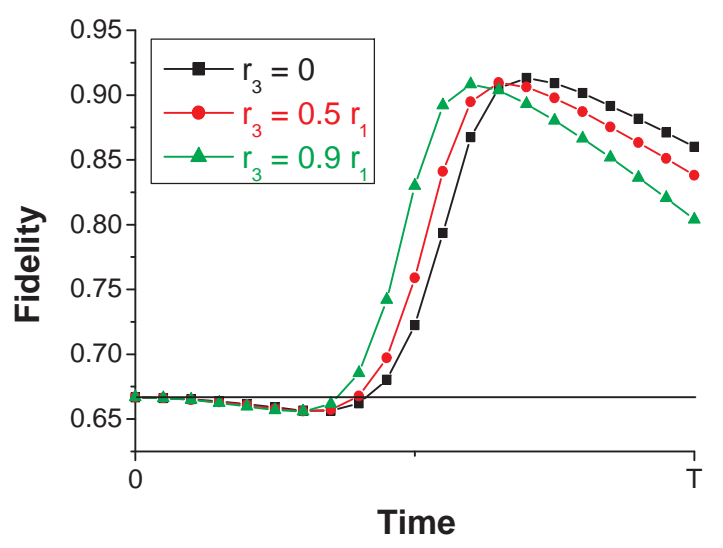

FIG. 1: (Color online.) Fidelity of the $\mathcal{H}$ gate, as a function of time: Effect of distributed noise. The fidelities are calculated for $r_{1}=10$, and $r_{2}=9.5$, for different values of $r_{3}$. The (parallel and transverse) fields for which the calculations are performed are depicted in Fig. 5 As seen in the figure, the maximal fidelities are obtained a little after $t=T / 2$. $T$ is a time that satisfies Eq. (3), which with our chosen parameters mean $T \gg 7 \times 10^{6} \hbar / \lambda$. Note that the fidelity does not change appreciably with the increase of the distributed noise level $r_{3}$. The local noise is assumed to absent (i.e. $\varepsilon=0$ ). The horizontal line at $2 / 3$ denotes the limit above which the gate fidelity is quantum.

$$
\begin{aligned}
|01\rangle \rightarrow\left|\psi^{+}\right\rangle & \equiv \frac{|01\rangle+|10\rangle}{\sqrt{2}}, \\
|10\rangle \rightarrow-\left|\psi^{-}\right\rangle & \equiv-\frac{|01\rangle-|10\rangle}{\sqrt{2}} .
\end{aligned}
$$

The gate is manifestly entangling, and we call it the Bell gate.

\section{THE GATE IMPLEMENTATION PROTOCOLS: ADIABATIC PASSAGE}

The second and final step of our quantum neural network model of quantum computation consists in the implementation of the quantum gates, by adiabatic passage of the whole system by adiabatic tuning of parallel and transverse magnetic fields.

\section{A. Protocol for the $\mathcal{H}$ gate}

Let us first consider the protocol for implementing the single qubit $\mathcal{H}$ gate. Note that in this case, the encoding is given by Eq. (12). To implement the $\mathcal{H}$ gate, a qubit that is initially in the state

$$
a_{0}|0\rangle+a_{1}|1\rangle
$$

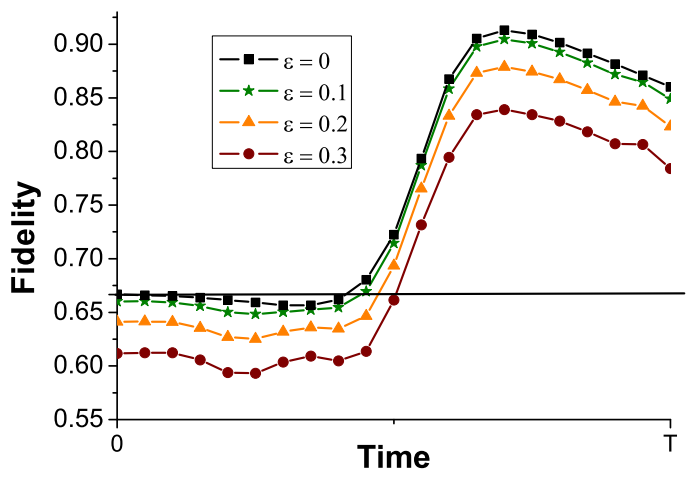

FIG. 2: (Color online.) Fidelity of the $\mathcal{H}$ gate, as a function of time: Effect of local noise. The fidelities are calculated for $r_{1}=10, r_{2}=9.5$, and $r_{3}=0$, for different values of $\varepsilon$. Just like in the case of distributed noise in Fig. 1 the (parallel and transverse) fields for which the calculations are performed are depicted in Fig. 5. And again, the maximal fidelities are obtained a little after $t=T / 2$, where $T$ is a time that satisfies Eq. (3), which with our chosen parameters mean $T \gg 7 \times 10^{6} \hbar / \lambda$. The fidelity does not show a marked diminish with the increase of the level of local noise $\varepsilon$. The distributed noise is assumed to be absent (i.e. $r_{3}=0$ ). The horizontal line at $2 / 3$ denotes the limit above which the gate fidelity is quantum. Note that the $\varepsilon=0$ curve in this figure is the same as the $r_{3}=0$ curve in Fig. 1]

(in the logical basis), should evolve into the state

$$
a_{0}|+\rangle-a_{1}|-\rangle \text {. }
$$

Here $a_{0}$ and $a_{1}$ are complex numbers, with $\left|a_{0}\right|^{2}+\left|a_{1}\right|^{2}=$ 1. Using the encoding in Eq. (12), the qubit is initially in the state

$$
a_{0}|G(0)\rangle+a_{1}\left|E_{1}(0)\right\rangle .
$$

We now adiabatically change the fields in the QNN Hamiltonian up to a certain time $t=T$, in which case, the system that was initially in the state in Eq. (22), evolves, in accordance with the adiabatic theorem, to the state

$$
a_{0} e^{i \Phi_{0}}|G(T)\rangle+a_{1} e^{i \Phi_{1}}\left|E_{1}(T)\right\rangle,
$$

where the phases $\Phi_{i}$ are given by the sums of the dynamical and Berry phases for the corresponding eigenstates [18, 19, 20, 21]. The eigenvectors of the Hamiltonian that appear in our calculations of the fidelities of the $\mathcal{H}$ gate as well as the Bell gate, are all real in at least one basis. Consequently, the corresponding Berry phases vanish. Therefore, the total phase is given by the dynamical phase:

$$
\Phi_{i}=\Phi_{i}^{D}=-\int_{0}^{T} E_{i}(t) d t, \quad i=0,1,2, \ldots
$$




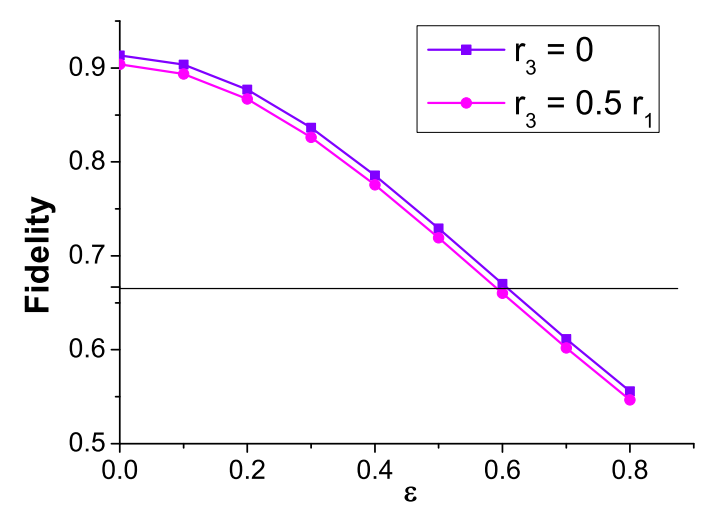

FIG. 3: (Color online.) Fidelity of the $\mathcal{H}$ gate: Combined effect of distributed and local noise. We plot the maximal fidelity of the $\mathcal{H}$ gate as a function of the local noise parameter $\varepsilon$, when the distributed noise parameter $r_{3}$ is vanishing and 0.5. The time for calculation of the fidelities is chosen to be the one for which maximal fidelity is obtained for $\varepsilon=0$ and $r_{3}=0$. Again the fields are changed as in Fig. 5. and adiabaticity requires that the time of fidelity calculation be $\gg 7 \times 10^{6} \hbar / \lambda$. The horizontal line at $2 / 3$ denotes the limit above which the gate fidelity is quantum.

Our aim is to change the fields in such a way that the final (time evolved) state in Eq. (23) is "as close as possible" to the $\mathcal{H}$ rotated state $a_{0}|+\rangle-a_{1}|-\rangle$, i.e. to

$$
a_{0} \frac{|G(0)\rangle+\left|E_{1}(0)\right\rangle}{\sqrt{2}}-a_{1} \frac{|G(0)\rangle-\left|E_{1}(0)\right\rangle}{\sqrt{2}} .
$$

The measure of closeness that we use is described in Subsect. VIC).

\section{B. Protocol for the Bell gate}

In the case of the Bell gate, the encoding is as in Eq. (13). Here, two qubits that are initially in the state

$$
a_{00}|00\rangle+a_{11}|11\rangle+a_{01}|01\rangle+a_{10}|10\rangle
$$

(in the logical basis), should evolve into the state

$$
a_{00}\left|\phi^{+}\right\rangle-a_{11}\left|\phi^{-}\right\rangle+a_{01}\left|\psi^{+}\right\rangle-a_{10}\left|\psi^{-}\right\rangle .
$$

Using the encoding in Eq. (13), the two qubits are initially in the state

$$
a_{00}|G(0)\rangle+a_{11}\left|E_{1}(0)\right\rangle+a_{01}\left|E_{2}(0)\right\rangle+a_{10}\left|E_{3}(0)\right\rangle .
$$

Again, adiabatic changes in the fields in the QNN Hamiltonian up to a certain time $t=T$, changes the state in Eq. (28) into the state

$$
\begin{aligned}
a_{00} e^{i \Phi_{0}}|G(T)\rangle+a_{11} e^{i \Phi_{1}}\left|E_{1}(T)\right\rangle & +a_{01} e^{i \Phi_{2}}\left|E_{2}(T)\right\rangle \\
& +a_{10} e^{i \Phi_{3}}\left|E_{3}(T)\right\rangle .
\end{aligned}
$$

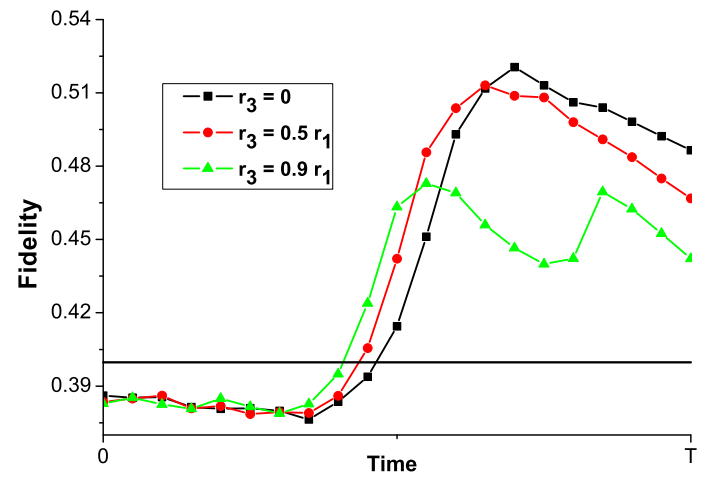

FIG. 4: (Color online.) Fidelity of the Bell gate, as a function of time. Just like in the case of the $\mathcal{H}$ gate in Fig. 1 the fidelities here are calculated for $r_{1}=10$, and $r_{2}=9.5$, and the fields are depicted in Fig. [5] As seen in the figure, for moderate values of the distributed noise $r_{3}$, the maximal fidelities are obtained around $t=3 T / 4$. The local noise is assumed to be absent here. The dip in the fidelity curve around $t=3 T / 4$ for the very high noise $\left(r_{3}=0.9 r_{1}\right)$ case, is due to the fact that the energy gap between the 1st excited state and the 2nd excited state becomes comparable to that between the 3rd and the 4th. Again, $T$ is a time that satisfies Eq. (3), which with our chosen parameters mean $T \gg 7 \times 10^{6} \hbar / \lambda$. There is no appreciable decrease in the fidelity upto about $r_{3}=0.5 r_{1}$. The horizontal line at $2 / 5$ denotes the limit above which the Bell gate fidelity is quantum.

Our strategy in this case is again to change the fields in such a way that the final (time evolved) state in Eq. (29) is as close as possible to the Bell rotated state

$$
\begin{array}{r}
a_{00} \frac{|G(0)\rangle+\left|E_{1}(0)\right\rangle}{\sqrt{2}}-a_{11} \frac{|G(0)\rangle-\left|E_{1}(0)\right\rangle}{\sqrt{2}} \\
+a_{01} \frac{\left|E_{2}(0)\right\rangle+\left|E_{3}(0)\right\rangle}{\sqrt{2}}-a_{10} \frac{\left|E_{2}(0)\right\rangle+\left|E_{3}(0)\right\rangle}{\sqrt{2}} .
\end{array}
$$

\section{Fidelity of a gate}

The fidelity $f$ of a gate is defined as the overlap between the required output state $|\Psi\rangle$ of the gate and the actual final state $\left|\Psi_{\text {out }}\right\rangle$, averaged over the Hilbert space of input states $|\psi\rangle$ :

$$
f=\int d(|\psi\rangle)\left|\left\langle\Psi \mid \Psi_{\text {out }}\right\rangle\right|^{2} .
$$

Note that both the ideally required output $|\Psi\rangle$, and the actual final state $\left|\Psi_{\text {out }}\right\rangle$, depends on the input state $|\psi\rangle$.

We compare the fidelities of our gates to the "classical" fidelity, a term which is commonly used in the following context: Suppose that a quantum gate takes a $d$ level quantum system as its input. Consider a situation where, instead of using the quantum gate, one uses the strategy 
of measuring the input (thus making the information in the quantum input as classical), and then preparing an output from the information obtained from the measurement on the input. The maximal fidelity that is obtainable in this way is said to be the classical fidelity of the gate. Note that the only parameter of the quantum gate that is used here is the dimension of the input space of the gate. The classical fidelity of a quantum gate that takes $d$ level systems at its input is (see e.g. 27])

$$
\frac{2}{d+1}
$$

\section{FIDELITIES OF THE $\mathcal{H}$ AND BELL GATES}

We now study the fidelities for the $\mathcal{H}$ and the Bell gates. Starting with the $\mathcal{H}$ gate, we investigate the behavior of the fidelity as a function of time, for an exemplary set of values of the parameters in the QNN Hamiltonian. We consider the influence of distributed noise in Fig. 1, whereas in Fig. 2 we show the fidelity for different levels of local noise. Notice that in both cases of noise, even substantial increases in the noise level do not change the fidelity very much. Moreover, there is a large region of the time axis where the fidelity is larger than the classical limit $2 / 3 \approx 0.667$.

We have so far considered the influences of local noise and distributed noise separately. In a more realistic scenario, however, the two sources of noise act simultaneously. We investigate this situation in Fig. 3. We note again that even substantially increasing both the noise levels does not affect the fidelity in a dramatic way.

The corresponding calculations for the Bell gate lead to qualitatively similar results. The values obtained for the fidelities, for exactly the same system parameters as for the $\mathcal{H}$ gate in Fig. [1 are displayed in Fig. 4. Note that the classical limit in this case is $2 / 5=0.4$.

The changes of the fields that we make for the above implementation of the gates are the same for both the gates, and are shown in Fig. 5 .

The gate fidelities as shown in Figs. $1,2,3$ and 4 are for the case when $r_{1} \approx r_{2} \gg r_{3}$, and as shown in Ref. [16, 25], the latter requirement cannot be met in a harmonic confinement of the ions. Many experimental strategies, however, consider a harmonic confinement, in which case one has $r_{1} \gg r_{2}, r_{3}$ [16, 25], and as we show in Fig. 6, one can implement a noise resistant $\mathcal{H}$ gate in such a trap.

Let us note here that in all the above figures, where fidelities of gates are plotted with respect to time, the curves for the fidelities have small curvatures at and around the positions of maximum fidelities. This implies that in an implementation of the presented protocols, small errors in the time of measurement (of the fidelity), does not affect the gate fidelities appreciably.

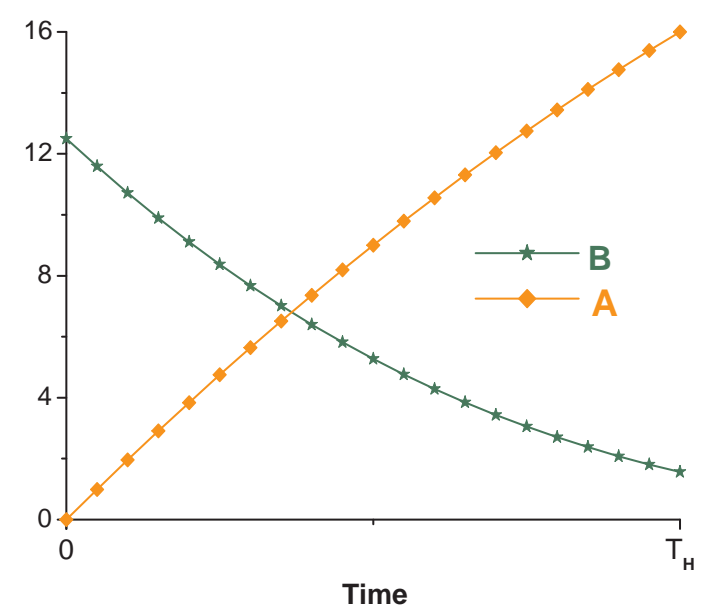

FIG. 5: (Color online.) The adiabatic change in the fields that effects the $\mathcal{H}$ and Bell gates as shown in Figs. 112 3 and 4. as implemented in a fountain trap, as well as in the implementation of the $\mathcal{H}$ gate in Fig. [6] in a harmonic trap. The fields are $A(t) \lambda$ and $B_{1}(t) \lambda=10^{-5} B(t) \lambda$ and $B_{2}(t) \lambda=$ $10^{-6} B(t) \lambda$, where $A(t)$ and $B(t)$ are as shown in the figure. For this choice of the fields, adiabaticity requires that $T_{H} \gg$ $7 \times 10^{6} \hbar / \lambda$. This time $T_{H}$ corresponds to the time at which the fidelity of the $\mathcal{H}$ gate, for $r_{3}=0$, attains its maximum.

\section{ADIABATICITY AND THE AVOIDED CROSSINGS}

The above calculations were performed by keeping in mind that we must respect the adiabaticity condition. As we have noted before, the adiabaticity condition demands that we should have

$$
T \gg \hbar \frac{\left\|\frac{d}{d s} H(s)\right\|}{g(s)^{2}} .
$$

For the case of the one-qubit gate considered, there are two energy levels involved. They are respectively the ground and the first excited state of the whole system (the QNN). In the case of the two-qubit gate we considered, there are four energy levels involved. They are the ground state, and the first, second and third excited states of the whole system. The maximal gate fidelities are reached after the system passes through a "double" avoided crossing. One of the avoided crossings is between the ground state and the first excited state, while the other is between the second and the third excited states. They appear almost at the same time. In Fig. 7. we show the dynamics of the five lowest energy eigenvalues, when $r_{1}=10, r_{2}=9.5$, and $r_{3}=0$, and the fields as in Fig. 5 A typical value for the energy gap at the avoided crossing is $\lambda \times 0.03$. Note that for the adiabatic transfer in the implementation of the $\mathcal{H}$ gate, the three lowest levels are the relevant ones, while for the Bell gate 


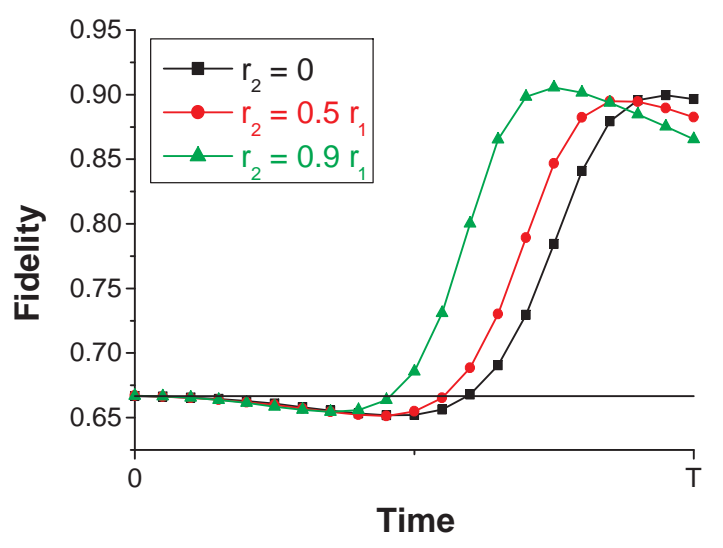

FIG. 6: (Color online.) Fidelity of the $\mathcal{H}$ gate, as a function of time, in a harmonic confinement. The fidelities are calculated for $r_{1}=10$, and the fields are as in Fig. 5 $T$ is a time that satisfies Eq. (3), which with our chosen parameters mean $T \gg 7 \times 10^{6} \hbar / \lambda$. Note that the distributed noise parameter is now $r_{2}$, in contrast to that in Figs. 1, 2, 3, and 4, The local noise is assumed to be absent. Again the fidelities do not change appreciably with the increase of the noise level $r_{2}$. The horizontal line at $2 / 3$ denotes the limit above which the gate fidelity is quantum.

implementation, the five lowest levels are relevant. For the above values of $r_{1}$ and $r_{2}$, and for values of $r_{3}$ up to $\approx 0.9 r_{1}$, the typical energy gap (at the avoided crossing), remains approximately at $\lambda \times 0.03$. For higher values of the distributed noise level $r_{3}$, i.e. for the case when $r_{1} \approx r_{2} \approx r_{3}$, this gap collapses, and hence it is no more possible to implement the gates in the presented way.

We denote by $T_{H}$, the point of time at which the maximal fidelity is reached for the $\mathcal{H}$ gate, for vanishing distributed noise $r_{3}$, and vanishing local noise $\varepsilon$, in the fountain trap. In the same situation, the maximal fidelity of the Bell gate is attained approximately at the same point of time. The avoided crossing is approximately at $3 T_{H} / 4$. Adiabaticity demands that

$$
T_{H} \gg 7 \times 10^{6} \frac{\hbar}{\lambda}
$$

\section{DISCUSSION}

We suggest a realization of universal quantum computing on an experimentally viable system of distributed qubits: The qubits are encoded in the (low) energy levels of the whole system. As in classical neural networks, where the distributed storage of classical information allows for robustness to noise, we show that our quantum system is resistant to high levels of noise. The one- and two-qubit quantum gates described in this paper are realized via adiabatic passage of the system from one set of

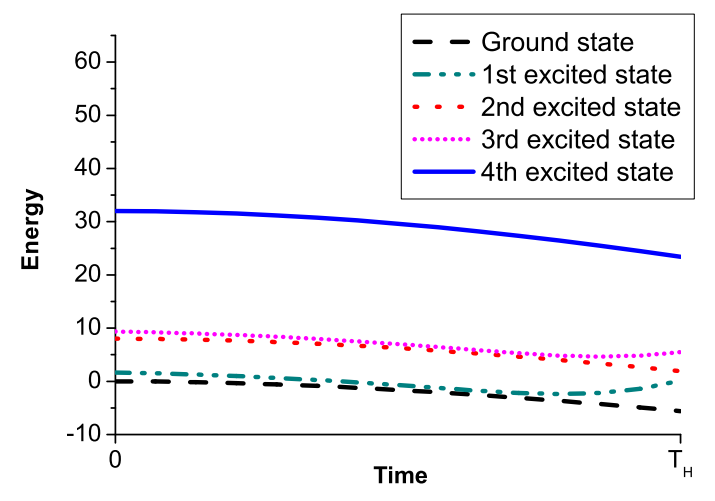

FIG. 7: (Color online.) Distribution of the five lowest energy levels for the time evolution (with the system parameters being just as in Fig. 1 with $r_{3}=0$ ), up to the point of maximal fidelity for the $\mathcal{H}$ gate in Fig. 1 (for $r_{3}=0$ ). The maximal fidelity for the Bell gate in Fig. 4 is obtained not long after that of the $\mathcal{H}$ gate in Fig. 1 Note that the energy gap between the ground state and first excited state, as well as that for the second excited and third excited state, are scaled up by a factor of 300 (in the figure), for better visibility. Also the actual energy gaps as shown in the figure are to be multiplied by $\lambda$, to have the correct unit and value.

energy eigenstates to another set of corresponding eigenstates. The adiabatic transfer is effected by a slow change of parallel and transverse fields. We perform numerical simulations to obtain the gate fidelities, and show that for a certain slow change of the fields, the gate fidelities are indeed much higher than their classical limits. We also observe that the fidelities typically have small curvatures near their maxima. Therefore, the gate fidelities will not change appreciably for small errors, in the time of measuring of the fidelities, in the experiments. The scalability issue is like in other proposals and experiments in ion-trap quantum computing [28], and may potentially be overcome by connecting mesoscopic clusters of trapped ions by flying qubits.

In this paper, we have considered the implementations of two gates: A one-qubit gate, which we have called the $\mathcal{H}$ gate, because of its similarity to the Hadamard gate, and a two-qubit gate, which we call the Bell gate, because the output states for an input computational basis, are the Bell states (up to phases). For the implementation of the $\mathcal{H}$ gate, there are two energy levels involved: the ground state and the first excited state of the whole system (the quantum neural network). For the implementation of the Bell gate, there are four energy levels involved: the ground state, and the first three excited states of the whole system. We observe that the maximal gate fidelities are reached after the system passes through a "double" avoided crossing. One of the avoided crossings is between the ground state and the first excited state, while the other is between the second and 
third excited states, and they appear almost at the same point of time. We find the condition under which the adiabaticity is realized.

The approach to noise management in both classical and quantum computational networks can broadly be divided into two categories: "active" and "passive" schemes. In a typical active noise-management scheme, the classical or quantum information is encoded in several bits or qubits, so that if noise occurs, it can be detected, and subsequently corrected. The active scheme is followed, for example, for data storage in a typical compact disc of our classical computers. There is also a corresponding theory of quantum error correction and fault tolerant quantum computation [3], that acts in an active way to correct errors. Typically, such a scheme will require encoding the quantum information into multiqubit entangled states, detecting any possible noise effect by multiqubit measurements, and reverting the effect of noise by a multiqubit unitary (which can be replaced by single-qubit and two-qubit unitaries).

A typical passive noise-management tries to identify a system that is already resistant to errors. Classical systems that manage noise effects in this way is are neural network models of brains. Examples of such noisemanagement in quantum circuits include the theories of decoherence-free subspaces [4] and topological quantum computation [7]. Our model of quantum computation by using a quantum neural network also falls into this category. A similarity between our scheme and a typical active noise-management scheme [3] is that both require to deal with several qubits: While the methods in Refs. [3] encode the quantum information in several qubits so that a possible noise effect is detectable and reversible, our quantum neural network model of quantum computation encodes the quantum information in a mesoscopic number of qubits so that the system is resilient towards noise. However, our model does not require encoding into multiqubit entangled states, or measurements onto multiqubit bases. Moreover, the step to revert the effect of noise is absent.

\section{Acknowledgments}

We acknowledge helpful comments from Jürgen Eschner, and support from the DFG (SFB 407, SPP 1078, SPP 1116), ESF QUDEDIS, Spanish MEC (FIS-200504627, Consolider Project QOIT, and Ramon y Cajal), EU IP SCALA, DAAD (German Academic Exchange Service), and the Ministry of Education of the Generalitat de Catalunya.
[1] P. Shor, SIAM J. Sci. Statist. Comput. 26, 1484 (1997) (quant-ph/9508027).

[2] L. Grover, in Proceedings of the 28th Annual ACM Symposium on the Theory of Computing (STOC), pp. 212219 (1996) (quant-ph/9605043); Phys. Rev. Lett. 79, 325 (1997).

[3] P. Shor, in 37th Symposium on Foundations of Computing, pp. 56-65 (IEEE Computer Society Press, 1996) (quant-ph/9605011); E. Knill and R. Laflamme, quant-ph/9608012 E. Knill, R. Laflamme and W. Zurek, quant-ph/9610011. Proc. R. Soc. Lond. A 454, 365 (1998) (quant-ph/9702058); D. Aharonov and M. BenOr, quant-ph/9611025 quant-ph/9906129 A.Y. Kitaev, in Proc. 3rd Int. Conf. of Quantum Communication and Measurement (Plenum, NY, 1997), and references therein.

[4] L.M. Duan and G.C. Guo, Phys. Rev. Lett. 79, 1953 (1997); P. Zanardi and M. Rasetti, Phys. Rev. Lett. 79, 3306 (1997); D.A. Lidar, I.L. Chuang, and K.B. Whaley, Phys. Rev. Lett. 81, 2594 (1998).

[5] E. Knill, R. Laflamme, and L. Viola, Phys. Rev. Lett. 84, 2525 (2000).

[6] L. Viola and S. Lloyd, Phys. Rev. A. 58, 2733 (1998); L. Viola, E. Knill, and S. Lloyd, Phys. Rev. Lett. 82, 2417 (1999); P. Zanardi, Phys. Lett. A 258, 77 (1999); D. Vitali and P. Tombesi, Phys. Rev. A 65, 012305 (2002).

[7] A.Y. Kitaev, Annals Phys. 303, 2 (2003) (quant-ph/9707021); M.H. Freedman, A. Kitaev, and Z. Wang, Commun. Math. Phys. 227, 587 (2002) (quant-ph/0001071); M.H. Freedman, M. Larsen, and Z. Wang, quant-ph/0001108; M.H. Freedman, quant- ph/0003128; M.H. Freedman, A. Kitaev, M.J. Larsen, and Z. Wang, quant-ph/0101025. C. Mochon, Phys. Rev. A 67, 022315 (2003); B. Douçot, M.V. Feigel'man, L.B. Joffe, and A.S. Ioselevich, Phys. Rev. B 71, 024505 (2005), and references therein.

[8] P. Zanardi and M. Rasetti, Phys. Lett. A 264, 94 (1999); J. Pachos, P. Zanardi, and M. Rasetti, Phys. Rev. A 61, 10305 (2000); J.A. Jones, V. Vedral, A. Ekert, and G. Castagnoli, Nature 403, 869 (2000); A. Ekert, M. Ericsson, P. Hayden, H. Inamori, J.A. Jones, D.K.L. Oi, and V. Vedral, J. Mod. Opt. 47, 2501 (2000), and references therein.

[9] D.J. Amit, Modeling Brain Functions: The World of Attractor Neural Networks (Cambridge University Press, Cambridge, 1989); M. Mézard, G. Parisi, and M.A. Virasoro, Spin Glass Theory and Beyond: An Introduction to the Replica Method and Its Applications (World Scientific, Singapore, 1987).

[10] T.M. Cover and J.A. Thomas, Elements of Information Theory (Wiley, New York, 1991).

[11] D. Leibfried, E. Knill, S. Seidelin, J. Britton, R.B. Blakestad, J. Chiaverini, D.B. Hume, W.M. Itano, J.D. Jost, C. Langer, R. Ozeri, R. Reichle, and D.J. Wineland, Nature 438, 639 (2005).

[12] H. Häffner, W. Hänsel, C.F. Roos, J. Benhelm, D. Chekal-kar, M. Chwalla, T. Körber, U.D. Rapol, M. Riebe, P.O. Schmidt, C. Becher, O. Gühne, W. Dür, and R. Blatt, Nature 438, 643 (2005).

[13] D. Porras and J.I. Cirac, Phys. Rev. Lett. 92, 207901 (2004).

[14] C. Wunderlich, in Laser Physics at the Limit (Springer, 
Heidelberg, 2002), p. 261 (quant-ph/0111158).

[15] M. Lewenstein, A. Sanpera, V. Ahufinger, B. Damski, A Sen(De), and U. Sen, Adv. in Phys. 56, 243 (2007) (cond-mat/0606771).

[16] M. Pons, V. Ahufinger, C. Wunderlich, A. Sanpera, S. Braungardt, A. Sen(De), U. Sen, and M. Lewenstein, Phys. Rev. Lett. 98, 023003 (2007).

[17] M. Born and V. Fock, Zeitschrift für Physik 51, 165 (1928).

[18] A. Messiah, Quantum Mechanics, (John Wiley \& Sons, New York, 1958).

[19] S. Pancharatnam, Proc. Indian Acad. Sci. 44, 247 (1956).

[20] M.V. Berry, Proc. R. Soc. Lond. A 392, 84 (1984).

[21] A. Shapere and F. Wilczek (Eds.), Geometric Phases in Physics (World Scientific, Singapore, 1998).

[22] E. Farhi and S. Gutmann, Phys. Rev. A 57, 2403 (1998).

[23] E. Farhi, J. Goldstone, S. Gutmann, and M. Sipser, quant-ph/0001106. W. van Dam, M. Mosca, and U. Vazirani, in Proceedings of the 42nd Annual Symposium on
Foundations of Computer Science, pp. 279-287 (2001) quant-ph/0206003).

[24] D. Aharonov, W. van Dam, J. Kempe, Z. Landau, S. Lloyd, and O. Regev, quant-ph/0405098

[25] M. Pons, V. Ahufinger, C. Wunderlich, A. Sanpera, and M. Lewenstein, cond-mat/0512606

[26] W. Dur, G. Vidal, and J. I. Cirac, Phys. Rev. A 62, 062314 (2000).

[27] M. Horodecki, P. Horodecki, and R. Horodecki, Phys. Rev. A 60, 1888 (1999).

[28] See e.g., S. Seidelin, J. Chiaverini, R. Reichle, J. J. Bollinger, D. Leibfried, J. Britton, J. H. Wesenberg, R. B. Blakestad, R. J. Epstein, D. B. Hume, W. M. Itano, J. D. Jost, C. Langer, R. Ozeri, N. Shiga, and D. J. Wineland, Phys. Rev. Lett. 96, 253003 (2006).

[29] D. Deutsch, A. Barenco, and A. Ekert, Proc. R. Soc. Lond. A 449, 669 (1995), and references therein. 